\title{
Renin-Angiotensin System Blockade Improves Cardiac Indices in Acromegaly Patients
}

\section{(c) (2) (1)}

\author{
Authors \\ Julia D. J. Thomas ${ }^{1}$, Abhishek Dattani1, 2, Filip Zemrak², Thomas Burchell2, Scott A. Akker³, Felicity J. L. Kaplan4, \\ Bernard Khoo ${ }^{5}$, Simon Aylwin', Ashley B. Grossman7, L. Ceri Davies², Márta Korbonits
}

\section{Affiliations}

1 Centre for Endocrinology, WHRI, Barts \& the London School of Medicine and Dentistry, Queen Mary University of London

2 NIHR Cardiovascular Biomedical Research Unit, St Bartholomew's Hospital, London

3 Department of Endocrinology, St Bartholomew's Hospital, London

4 Department of Endocrinology, Lister Hospital, Stevenage, Herts

5 Department of Endocrinology, Royal Free Campus, UCL Medical School, London

6 Department of Endocrinology, King's College Hospital, London

7 Oxford Centre for Endocrinology, Diabetes and Metabolism, University of Oxford

Key words pituitary, acromegaly, cardiac magnetic resonance, reninangiotensin

received 23.07.2016

revised $\quad 07.12 .2016$

accepted 12.12.2016

\section{Bibliography}

DOI http://dx.doi.org/10.1055/s-0042-123710

Published online: 6.2.2017

Exp Clin Endocrinol Diabetes 2017; 125: 365-367

(c) J. A. Barth Verlag in Georg Thieme Verlag KG Stuttgart .

New York

ISSN 0947-7349

Correspondence

Márta Korbonits, MD, PhD

Professor of Endocrinology and Metabolism
Centre for Endocrinology, William Harvey Research Institute Barts and The London School of Medicine Queen Mary University of London Charterhouse Square EC1M 6BQ, London

UK

Tel.: + 44/20/7882 6238

korbonits@qmul.ac.uk

\section{ABSTRACT}

Purpose Blockade of the angiotensin-renin system, with angiotensin converting enzyme inhibitors (ACEi) and angiotensin receptor blockers (ARBs), has been shown to improve cardiac outcomes following myocardial infarction and delay progression of heart failure. Acromegaly is associated with a disease-specific cardiomyopathy, the pathogenesis of which is poorly understood.

Methods The cardiac indices of patients with active acromegaly with no hypertension (Group $A, n=4$ ), established hypertension not taking ACEi/ARBs (Group $B, n=4$ ) and established hypertension taking ACEi/ARBs (Group C, $n=4$ ) were compared using cardiac magnetic imaging.

Results Patients taking ACEi/ARBs had lower end diastolic volume index (EDVi) and end systolic volume index (ESVi) than the other 2 groups ([C] 73.24 vs. [A] 97.92 vs. [B] $101.03 \mathrm{ml}$ $m^{2}$, ANOVA $p=0.034$, B vs. $\left.C p<0.01\right)$. Groups A and B had EDVi and ESVi values at the top of published reference range values; Group $C$ had values in the middle of the range.

Conclusion Acromegaly patients on ACEi/ARBs for hypertension demonstrate improved cardiac indices compared to acromegaly patients with hypertension not taking these medications. Further studies are needed to determine if these drugs have a beneficial cardiac effect in acromegaly in the absence of demonstrable hypertension.

\section{Introduction}

Blockade of the renin-angiotensin system, using angiotensin converting enzyme inhibitors (ACEi) and angiotensin receptor blockers (ARBs), has been shown to reduce left ventricle (LV) remodeling in patients with myocardial infarction and delay the disease pro- gression in heart failure [1-3]. Patients with growth hormone $(\mathrm{GH})$ excess (acromegaly) develop a disease-specific cardiomyopathy associated with biventricular hypertrophy and diastolic dysfunction, that progresses to fulminant cardiac failure if left untreated [4-6]. In this preliminary study we compared the cardiac indices of $12 \mathrm{pa}$ - 
tients with active acromegaly, 4 of whom had no diagnosis of hypertension, 4 with an established diagnosis of hypertension on ACEi or ARBs and 4 with an established diagnosis of hypertension not taking these classes of drugs. Cardiac indices were compared using cardiac magnetic resonance imaging (CMR).

\section{Material and Methods}

12 patients with active acromegaly (clinical features, raised insulin-like growth factor 1 [IGF1] and failure to suppress GH on oral glucose tolerance testing) who were about to commence therapy underwent CMR to assess cardiac function and morphology: LV mass index (LVMi); end diastolic volume index (EDVi); end systolic volume index (ESVi); ejection fraction (EF). Measurements were indexed to body surface area. CMR was performed on a Philips Achieva CV 1.5 Tesla MRI scanner (Philips Medical Systems, Guildford, UK). Standard protocols were used. In this observational study, patients were divided into 3 groups: those with no known history of hypertension (A); those with a known diagnosis of hypertension not currently taking $A C E i / A R B(B)$ and those with a known diagnosis of hypertension taking ACEi/ARB (C). Groups $B$ and $C$ patients may also have been taking additional antihypertensive medication ( $\triangleright$ Table 1). Weight, height and blood pressure, serum IGF1 and fasting glucose levels were recorded. Patients with known ischaemic heart disease or heart failure were excluded. Multi-centre ethical approval was given by the local Research Ethics Committee (06/Q0401/53). Statistics were calculated using ANOVA with Bonferroni correction.

\section{Results}

Patient characteristics are given in $>$ Table 2, with no significant difference in IGF-1, blood pressure and fasting glucose. Group C patients were older and tended to have higher baseline blood pressure values than the other 2 groups. There was no difference in LVMi between the 3 groups. No patient had evidence of frank heart fail-

- Table 1 List of anti-hypertensives patients were on.

\begin{tabular}{|l|l|c|l|}
\hline \multicolumn{2}{|l|}{ Group B } & \multicolumn{2}{c|}{ Group C } \\
\hline Patient & Anti-hypertensives & Patient & Anti-hypertensives \\
\hline 5 & Bendrofluazide & 3 & Ramipril \\
\hline 7 & $\begin{array}{l}\text { Amiloride } \\
\text { Cyclopenthiazide }\end{array}$ & 4 & $\begin{array}{l}\text { Ramipril } \\
\text { Bendrofluazide }\end{array}$ \\
\hline 9 & Atenolol & 10 & Ramipril \\
\hline 11 & None & 12 & $\begin{array}{l}\text { Losartan } \\
\text { Amlodipine } \\
\text { Doxazosin }\end{array}$ \\
\hline
\end{tabular}

ure (all had an EF within normal limits). Patients in Group C had lower EDVi and ESVi than the other 2 groups ( $>$ Fig. 1). Patients in Groups A and B had EDVi and ESVi values at the top of published reference range values. Patients in Group $C$ had values in the middle of the reference range [7].

\section{Discussion}

This preliminary study indicated that patients taking ACEi/ARBs had lower end diastolic volume index (EDVi) and end systolic volume index (ESVi) than the other 2 groups including patients with no hypertension or hypertension treated with other drugs. While patients in group $C$ were older and tended to have a higher mean blood pressure than groups A and B, this, if anything, should have increased the prevalence of cardiac remodeling in this group whereas the converse was seen with reduced remodeling, adding more evidence to the suggestion that ACEi/ARBs were influential in reducing EDVi and ESVi.

EDVi and ESVi are markers of cardiac remodeling, the process of change in size, shape and function of the heart, arising from cardiac load or injury [8]. As this process continues, heart failure develops and a reduction in $\mathrm{EF}$ is seen. ACEi and ARBs have been shown to delay progression of ischaemic heart failure and to improve survival through prevention and possible reversal of this remodeling process $[1,3,9,10]$.

The reasons for the improvement in LV EDVi and ESVi in patients treated with ACEi/ARBs are likely to be multifactorial. Firstly, acromegalic cardiomyopathy appears to be more marked in patients who have concurrent hypertension [11]. Additionally, acromegaly may be associated with increased serum aldosterone levels [12]. Blockade of the renin-angiotensin pathway will reduce the effects of aldosterone excess, reducing cardiac pre-load and possibly local myocardial response to aldosterone excess, thought to play a part in promoting remodeling [8]. Furthermore, it has been found that ACEi can attenuate the ability of IGF1 to produce cardiac fibroblast proliferation, indicating that these medications may have a direct effect on the response of the myocardium to $\mathrm{GH}$ action, directly inhibiting the development of acromegalic cardiomyopathy [13].

This study did not demonstrate any reduction in LVMi associated with ACEi/ARB use, although this may be related to group size. Given the changes seen in EDVi and ESVi, and the lower mean LVMi in group $C$ compared to group $B$, it would be interesting to address this in a larger study.

It should be noted that ACEi are not more efficacious in reducing cardiovascular morbidity or mortality than other forms of anti-hypertensive medications in patients without acromegaly [14]. However, the changes seen in this preliminary study suggest that patients with acromegaly may derive benefits from these medications over and above those arising simply from the normalisation of hypertension.

- Table 2 Patient characteristics. Data given as group mean and [SD]. BP - blood pressure.

\begin{tabular}{|c|c|c|c|c|}
\hline & Group A $(n=4)$ & Group B $(n=4)$ & Group C $(n=4)$ & P-value \\
\hline Number of females & $2 / 4$ & $3 / 4$ & $3 / 4$ & $\mathrm{~N} / \mathrm{A}$ \\
\hline Age (years) & $41.8[7.6]$ & $54.5[10.6]$ & $62.8[11.2]$ & 0.043 \\
\hline IGF1 (SDS) & $11.1[5.5]$ & $19.0[17.2]$ & $14.4[11.1]$ & 0.669 \\
\hline Systolic BP $(\mathrm{mmHg})$ & $125[26]$ & $133[23]$ & $147[15]$ & 0.392 \\
\hline Diastolic BP (mmHg) & 74 [17] & $76[12]$ & $82[6]$ & 0.655 \\
\hline Fasting Glucose (mmol/L) & $5.5[0.82]$ & $5.1[0.44]$ & $5.8[1.13]$ & 0.483 \\
\hline
\end{tabular}



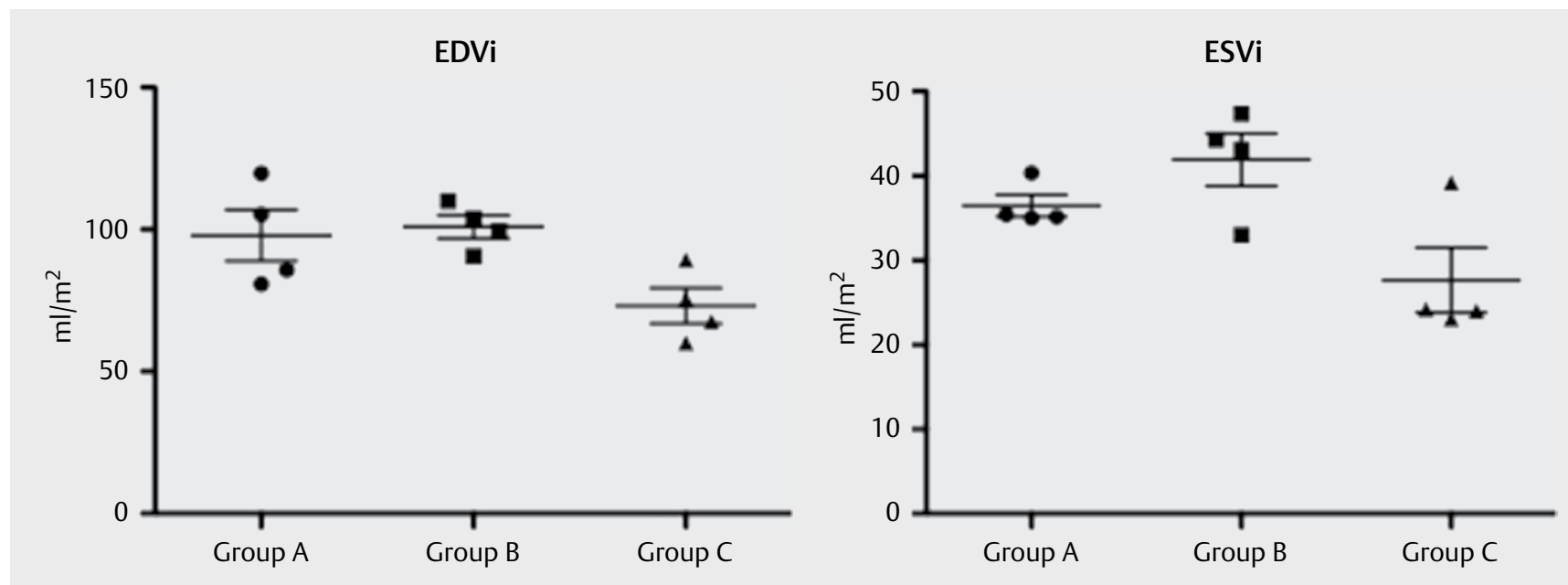

- Fig. 1 Graph comparing EDVi and ESVi in the 3 groups. There is a significant difference $(p<0.01)$ between Group B and Group C. Error bars indicate SEM.

The main limitation of this study is the small sample size. We note that the study was open to several large UK centres for 3 years and this was the number of acromegaly patients who fulfilled inclusion criteria and agreed to take part in the study. In such a rare condition, greater number of patients is often difficult to achieve. Albeit a small sample, these are the first data suggesting that ACEi/ ARBs may have a significant benefit in acromegaly patients over and above normalisation of blood pressure.

\section{Conclusion}

In this preliminary study, acromegaly patients with hypertension taking ACEi/ARBs demonstrated evidence of less cardiac remodeling than those taking alternative anti-hypertensives. We suggest that these agents should be considered first line anti-hypertensives in patients with this diagnosis. Larger studies are needed to confirm these findings and determine if there is a role using these medications in patients with active acromegaly who have not yet developed hypertension.

\section{Acknowledgements}

This work forms part of the research themes contributing to the translational research portfolio of Barts Cardiovascular Biomedical Research Unit, which is supported and funded by the National Institute for Health Research. The study was supported by unrestricted research grants from Pfizer and Novartis. Declaration of interests: MK is a member of the Scientific Advisory Board of Pfizer.

\section{Conflict of Interest}

None.

\section{References}

[1] Suzuki H, Kusuyama T, Omori Y et al. Inhibitory effect of candesartan cilexetil on left ventricular remodeling after myocardial infarction. Int Heart J 2006; 47: 715-725
[2] Bazzino O, Navarro Estrada JL, Sosa Liprandi A et al. Early treatment with low-dose enalapril after acute myocardial infarction: an equilibrium radionuclide angiographic study. Enalapril despues del Infarto (EDI) Trial Investigators. J Nucl Cardiol 1997; 4: 133-139

[3] Greenberg B, Quinones MA, Koilpillai C et al. Effects of long-term enalapril therapy on cardiac structure and function in patients with left ventricular dysfunction. Results of the SOLVD echocardiography substudy. Circulation 1995; 91: 2573-2581

[4] Sacca L, Cittadini A, Fazio S. Growth hormone and the heart. Endocr Rev 1994; 15: 555-573

[5] Colao A, Marzullo P, Di Somma C et al. Growth hormone and the heart. Clin Endocrinol 2001; 54: 137-154

[6] Clayton RN. Cardiovascular function in acromegaly. Endocr Rev 2003; 24: 272-277

[7] Kawel-Boehm N, Maceira A, Valsangiacomo-Buechel ER et al. Normal values for cardiovascular magnetic resonance in adults and children. Cardiovasc Magn Reson 2015; 17: 29

[8] Cohn JN, Ferrari R, Sharpe N. Cardiac remodeling-concepts and clinical implications: A consensus paper from an International Forum on Cardiac Remodeling. J Am Coll Cardiol 2000; 35: 569-582

[9] Konstam MA, Rousseau MF, Kronenberg MW et al. Effects of the angiotensin converting enzyme inhibitor enalapril on the long-term progression of left ventricular dysfunction in patients with heart failure. SOLVD Investigators. Circulation 1992; 86: 431-438

[10] Konstam MA, Kronenberg MW, Rousseau MF et al. Effects of the angiotensin converting enzyme inhibitor enalapril on the long-term progression of left ventricular dilatation in patients with asymptomatic systolic dysfunction. SOLVD (Studies of Left Ventricular Dysfunction) Investigators. Circulation 1993; 88: 2277-2283

[11] Fazio S, Cittadini A, Sabatini D et al. Evidence for biventricular involvement in acromegaly: a Doppler echocardiographic study. Eur Hear J 1993; 14: 26-33

[12] Marks P, Vincent R, Wilson B et al. Aldosterone in acromegaly. Am J Med Sci 1984; 287: 16-19

[13] van Eickels $M$, Vetter $H$, Grohé $C$. Angiotensin-converting enzyme (ACE) inhibition attenuates insulin-like growth factor-I (IGF-I) induced cardiac fibroblast proliferation. Br J Pharmacol 2000; 131: 1592-1596

[14] Hansson L, Lindholm LH, Niskanen L et al. Effect of angiotensin-converting-enzyme inhibition compared with conventional therapy on cardiovascular morbidity and mortality in hypertension: the Captopril Prevention Project (CAPPP) randomised trial. Lancet 1999; 353: 611-616 